\title{
Reduction by the positive allosteric modulator of the GABA receptor, GS39783, of alcohol self-administration in Sardinian alcohol-preferring rats exposed to the "sipper" procedure
}

\section{Paola Maccioni ${ }^{1+}$, Paolo Flore ${ }^{2 \dagger}$, Mauro A. M. Carai' , Claudia Mugnaini ${ }^{3}$, Serena Pasquini ${ }^{3}$, Federico Corelli ${ }^{3}$, Gian Luigi Gessa ${ }^{1}$ and Giancarlo Colombo ${ }^{1 *}$}

\author{
Consiglio Nazionale delle Ricerche Neuroscience Institute, Cagliari, Italy \\ 2 Department of Neuroscience, University of Cagliari, Cagliari, Italy \\ ${ }^{3}$ Department of Pharmaceutical and Applied Chemistry, University of Siena, Siena, Italy
}

Edited by:

Lorenzo Leggio, Brown University, USA

Reviewed by:

Malgorzata Filip, Polish Academy of

Sciences, Poland

Maria E. Quintanilla, Universidad de

Chile, Chile

*Correspondence:

Giancarlo Colombo, Consiglio

Nazionale delle Ricerche Neuroscience

Institute, Viale Diaz 182, I-09126,

Cagliari, Italy.

e-mail:colomb@unica.it

tPaola Maccioni and Paolo Flore are contributed equally to the work.
The present study was designed to evaluate (a) alcohol self-administration behavior of selectively bred, Sardinian alcohol-preferring (sP) rats exposed to the so-called "sipper" procedure (characterized by the temporal separation between alcohol-seeking and -taking phases), and (b) the effect of the positive allosteric modulator of the GABA ${ }_{B}$ receptor, GS39783, on alcohol self-administration in $\mathrm{SP}$ rats exposed to this procedure. To this end, $\mathrm{SP}$ rats were initially trained to lever-respond under a reinforcement requirement (RR) 55 (RR55) for alcohol. Achievement of RR55 resulted in the 20-min presentation of the alcohol $(15 \%, v / v)$-containing sipper bottle. Once stable levels of lever-responding and alcohol consumption were reached, rats were treated with 0, 25, 50, and $100 \mathrm{mg} / \mathrm{kg}$ GS39783 (i.g.) 60 min before the self-administration session. Rats displayed robust alcohol-seeking (as suggested by relatively short latencies to the first lever-response and high frequencies of lever-responding) and -taking (as suggested by alcohol intakes averaging approximately $1.5 \mathrm{~g} / \mathrm{kg}$ ) behaviors. Pretreatment with GS39783 inhibited both alcohol-seeking (the number of rats achieving RR55 and the mean RR value were virtually halved) and -taking (the amount of self-administered alcohol was reduced by approximately $60 \%)$. The results of the present study suggest the power of the "sipper" procedure in triggering high levels of alcohol-seeking and -taking behavior in SP rats. Further, these results extend to this additional procedure of alcohol self-administration the capacity of GS39783 to reduce the motivational properties of alcohol and alcohol consumption in SP rats.

Keywords: "sipper" procedure of alcohol self-administration, alcohol-seeking and -taking behavior, positive allosteric modulator of the GABA ${ }_{B}$ receptor, GS39783, Sardinian alcohol-preferring (sP) rats

\section{INTRODUCTION}

GS39783 (N,N'-dicyclopentyl-2-methylsulfanyl-5-nitro-pyrimidine4,6-diamine) is one of the few effective in vivo positive allosteric modulators (PAMs) of the $\mathrm{GABA}_{\mathrm{B}}$ receptor $\left(\mathrm{GABA}_{\mathrm{B}}\right.$ PAMs) synthesized to date (Urwyler et al., 2003; Cryan et al., 2004). Recent experimental data indicate that its administration specifically reduces operant, oral alcohol self-administration in selectively bred, Sardinian alcoholpreferring (sP) rats (Maccioni et al., 2007, 2008). These data are in line with the results of recent studies indicating the capacity of (a) GS39783 to reduce other alcohol-related behaviors, including alcohol drinking (Orrù et al., 2005), and (b) the other presently available $\mathrm{GABA}_{\mathrm{B}}$ PAMs, CGP7930, BHF177, and rac-BHFF to reduce operant, oral alcohol self-administration in sP and Indiana alcohol-preferring (P) rats (Liang et al., 2006; Maccioni et al., 2009, 2010). These data are also consonant with multiple lines of experimental and clinical evidence suggesting a role for the $\mathrm{GABA}_{\mathrm{B}}$ receptor system in the mediation of the behavioral and pharmacological effects of alcohol (see Maccioni and Colombo, 2009; Leggio et al., 2010).

The above-mentioned self-administration studies testing GS39783 and all other $\mathrm{GABA}_{\mathrm{B}}$ PAMs have been conducted using the two most conventional procedures of operant alcohol self-administration: the fixed ratio (FR) schedule of reinforcement and the progressive ratio (PR) schedule of reinforcement (see Markou et al., 1993). In the FR procedure, the response requirement (RR) - i.e., the "cost" of each alcohol presentation in terms of number of behavioral responses (lever-pressing, nose-poking, or spout-licking) - is kept fixed throughout the session; in other words, each alcohol access occurs after the animal has emitted the specifically required behavior [e.g., four responses on the lever in the study testing GS39783 (Maccioni et al., 2007)]. In the PR procedure, RR - likewise, the behavior needed to access each alcohol presentation - is progressively increased over the session [e.g.: 4, 9, $12,15,20,25,32,40,50,62,77,95,118,145,178,219$, and so on in the study testing GS39783 (Maccioni et al., 2008)]; the lowest ratio not completed or the highest ratio completed (defined as "breakpoint") is taken as the measure of alcohol's motivational properties. In both FR and PR procedures, alcohol presentations are (a) multiple over each single session, (b) of fixed and relatively small magnitude $(0.1 \mathrm{ml}$ is the common volume of each access), and (c) interspersed among sequences of operant behavior (i.e., animals alternate short periods during which they emit the operant behavior with short periods of alcohol consumption). Because of this latter feature, these 
procedures do not allow the appetitive, or motivational, aspects to be completely distinguished from the merely consummatory aspects of animal behavior. When these procedures are applied to pharmacological investigations, it is not always totally clear whether the tested drug is acting exclusively, or mutually, on the motivational or consummatory aspects of the animal behavior.

To possibly overcome this limitation, several years ago Dr. Herman H. Samson and his colleagues conceived and developed a procedure of operant, oral alcohol self-administration based on two temporally distinct phases: an initial, single phase of operant behavior, during which rats had to reach a given RR (representing the "alcohol-seeking" phase), and a subsequent, single phase of unlimited consumption of alcohol ("alcohol-taking" phase); of course, only rats achieving the assigned RR could access alcohol (Samson et al., 2000). This procedure was named "sipper" procedure because of the use of large bottles (sippers) that substituted the small-volume devices commonly used in most FR and PR studies. Subsequent pharmacological studies apparently confirmed the hypothesized capacity of this procedure to provide distinct sets of information on the effect of the tested drug on the appetitive and consummatory aspects of rat behavior (e.g.: Czachowski et al., 2001, 2006; Freedland et al., 2001; Sharpe and Samson, 2002; Slawecki and Roth, 2003).

The present study was therefore designed to extend to the "sipper" procedure the investigation on the reducing effect of GS39783 on operant, oral alcohol self-administration in rats. The present study was conducted using rats of the sP line, in which alcohol self-administration under the FR and PR schedules of reinforcement resulted as being highly sensitive to the suppressing effect of different $\mathrm{GABA}_{\mathrm{B}}$ receptor agents, including the direct agonist,

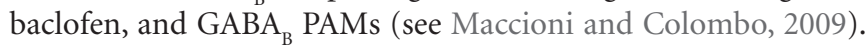
Implicitly, the present study was also aimed at testing the "sipper" procedure in $\mathrm{sP}$ rats, with the intent of assessing the potentiality of this procedure to unravel alcohol-seeking and -taking behavior in $\mathrm{sP}$ rats.

\section{MATERIALS AND METHODS}

The experimental procedures employed in the present study were in accordance with the Italian Law on the "Protection of animals used for experimental and other scientific reasons".

\section{ANIMALS}

Male sP rats $(n=12)$ from the 69th generation and 60-days-old at the start of the study, were utilized. Rat body weight averaged approximately 380 and $610 \mathrm{~g}$ at the start and at the end of the study, respectively. Rats were housed four per cage in standard plastic cages with wood chip bedding throughout the study. The animal facility was under an inverted 12:12-h light-dark cycle (lights on at 7:00 p.m.), at a constant temperature of $22 \pm 2{ }^{\circ} \mathrm{C}$ and relative humidity of approximately $60 \%$. Rats were extensively habituated to handling and intragastric infusions (by metal gavage). Food pellets (Mucedola, Settimo Milanese, Italy) and water were always available in the homecage, except as noted.

\section{APPARATUS}

Operant sessions were conducted in modular chambers (Med Associates, St. Albans, VT, USA), located in sound-attenuated cubicles, with fans for ventilation and background white noise. The front panel of each chamber was equipped with the retractable spout of a liquid sipper (250-ml capacity) located outside the chamber, and one white stimulus light mounted above the hole through which the sipper spout was made available. The back panel of each chamber was equipped with one retractable response-lever and one white stimulus light mounted above the lever. A white house light was centered at the top of the back panel of each chamber. Start of the session was signaled by illumination of the house light and insertion of the lever. Each lever-response was associated to flashing of the stimulus light mounted above the lever. Achievement of RR resulted in retraction of the lever, exposure of the sipper spout, and illumination of the stimulus light mounted above the spout hole for the period of exposure of the sipper spout. End of the session was associated to retraction of the sipper spout and switch off of stimulus light and house light. The sipper apparatus was equipped with a lickometer device that permitted the recording of the rat's contacts (licks) with the sipper spout.

\section{EXPERIMENTAL PROCEDURE}

Rats were initially exposed to the homecage two-bottle "alcohol versus water" choice regimen with unlimited access for $24 \mathrm{~h} /$ day over 10 consecutive days. The alcohol solution was presented at a concentration of $10 \%(\mathrm{v} / \mathrm{v})$. This initial phase was conducted to allow the rats to become accustomed to the taste of alcohol and start to experience its pharmacological effects, in order to possibly shorten the subsequent shaping phase of the operant procedure.

Immediately after the two-bottle choice regimen, the selfadministration procedure was started. In each of its phases (shaping, training, maintenance, and testing), self-administration sessions were conducted 5 days per week (Monday to Friday) during the first 4-6 h of the dark phase of the light/dark cycle.

Rats were deprived of water during the $18 \mathrm{~h}$ before the first six daily sessions in the operant chamber.

\section{Shaping phase}

This initial phase lasted 5 days. In the initial daily session (Session 1; lasting $120 \mathrm{~min}$ ), the sipper spout was exposed into the chamber. Sipper presentation occurred repeatedly and uncontingently in accordance with an unpredictable schedule. The sipper was filled with water. This session was conducted in order to accustom rats to the noise of the retractable sipper device and to the availability of the sipper as a source of fluids. Starting from Session 2, the lever was presented. Sessions 2-5 lasted $30 \mathrm{~min}$ and were conducted under an FR1 schedule of reinforcement for water. Specifically, each lever-response resulted in the sipper spout presentation for 20 and $10 \mathrm{~s}$ in Sessions 2-3 and 4-5, respectively.

\section{Training phase}

Sessions 6-13 lasted $30 \mathrm{~min}$. In Session 6, rats were exposed to an FR1 schedule of reinforcement for $10 \%(\mathrm{v} / \mathrm{v})$ alcohol. The number of reinforcements was limited to 40 . This limit was imposed to avoid possible overdoses of alcohol as rats were still water-deprived. Session 7 was conducted under the FR1 schedule of reinforcement for $10 \%(\mathrm{v} / \mathrm{v})$ alcohol with no limit in the number of reinforcements as water-deprivation was abolished. In Sessions 8 and 9, the alcohol concentration was increased to $15 \%(\mathrm{v} / \mathrm{v})$ (this concentration was subsequently maintained throughout the entire study). In Sessions 10-13, FR was increased to FR2 and FR4. 
In the subsequent sessions, the RR procedure was finally introduced. Specifically, rats were required to achieve RR within $20 \mathrm{~min}$; failure to complete RR within 20 min resulted in lever retraction and no sipper presentation. RR was gradually increased from RR4 to RR55 over 19 consecutive sessions (Sessions 14-34). Specifically, RR was increased from RR4 to RR8, RR12, RR17, RR22, RR28, RR34, RR41, RR48, and RR55; each RR increase occurred every other day. Achievement of RR resulted in sipper presentation for a fixed time of $20 \mathrm{~min}$. RR55 was selected as the final RR on the basis of preliminary data suggesting it was the highest $R R$ value achieved by all tested sP rats (this laboratory, unpublished results).

\section{Maintenance phase}

All 15 sessions of the maintenance phase (Sessions 35-49) were identical to the last session of the training phase (Session 34). Specifically: RR55, 20-min maximal time for RR achievement, 20-min sipper exposure, and 15\% (v/v) alcohol.

\section{Testing phase}

Test sessions were identical to those of the maintenance phase. Test sessions were conducted on Fridays; four regular maintenance sessions (Monday to Thursday) elapsed between test sessions. All doses of GS39783 were tested in each rat under a Latin-square design. GS39783 (synthesized in house by CM, SP, and FC) was suspended in $2 \mathrm{ml} / \mathrm{kg}$ distilled water with a few drops of Tween-80, and administered by gavage at the doses of $0,25,50$, and $100 \mathrm{mg} /$ $\mathrm{kg} 60 \mathrm{~min}$ before the start of the session. GS39783 dose-range, pretreatment time, and route of administration were identical to those used in previous studies on alcohol self-administration (Maccioni et al., 2007, 2008).

\section{MEASURED VARIABLES AND DATA ANALYSIS}

Alcohol-seeking phase-Measured variables were (a) RR achieved and (b) latency (expressed in s) to the first response on the lever (those rats that failed to press the lever were assigned the value of $1200 \mathrm{~s}$ ).

Alcohol-taking phase - Measured variables were (a) amount of self-administered alcohol [expressed in $\mathrm{g} / \mathrm{kg}$ pure alcohol, and determined weighing the sipper bottle (0.1-g accuracy) before and after the session; rats that did not achieve RR55 - and therefore did not access the sipper - were assigned the value of $0 \mathrm{~g} / \mathrm{kg}$ ] and (b) total number of licks on the sipper spout (rats that did not achieve RR55 were assigned the value of 0 ).

Data on the effect of GS39783 on each of the above variables were statistically analyzed by separate one-way ANOVAs for repeated measures, followed by the Newman-Keuls test for post hoc comparisons. Data on the effect of GS39783 on the number of rats achieving RR55 were analyzed by the Chi-square test.

\section{RESULTS}

All rats acquired and steadily maintained alcohol self-administration under the tested procedure. An illustration of rats' behavior during both alcohol-seeking and -taking phases is given by data collected in vehicle-treated animals in the sessions testing GS39783. Specifically, all rats displayed a relatively short latency to the first lever-response $[69.7 \pm 26.6 \mathrm{~s}$ (mean \pm SEM) $]$ and a relatively high frequency of lever-responding $[16.8 \pm 1.7$ responses/min (mean \pm SEM) $]$; all rats achieved RR55, therefore gaining access to the alcohol solution. Once the alcohol solution was available, vehicle-treated rats immediately started to lick the sipper spout [latency to the first lick: $1.5 \pm 0.1 \mathrm{~s}$ $($ mean \pm SEM) $]$ and consumed large amounts of alcohol $[1.5 \pm 0.1 \mathrm{~g} /$ $\mathrm{kg}($ mean \pm SEM $)]$ over the 20 -min session.

Pretreatment with GS39783 resulted in inhibition of the rats' alcohol-seeking behavior. Specifically, only 7/12, 7/12, and 6/12 rats treated with 25,50 , and $100 \mathrm{mg} / \mathrm{kg}$ GS39783, respectively, achieved RR55 $(P<0.05$, Chi-square test $)$. Additionally, pretreatment with GS39783 produced a decrease in the value of achieved $\operatorname{RR}[F(3,33)=2.91, P<0.05]$ (Figure 1, top panel); with respect to vehicle-treated rats, $R$ R achieved by rats treated with all three doses of GS39783 was reduced by 35-45\%.Pretreatment with GS39783 resulted in a tendency toward an increase in latency to the first response on the lever $[F(3,33)=2.17, P>0.05]$ (Figure 1, bottom panel).

Pretreatment with GS39783 was also associated with an inhibition of rats' alcohol-taking behavior. Specifically, pretreatment with GS39783 produced a reduction in the amount of self-administered alcohol $[F(3,33)=8.66, P<0.0005]$ (Figure 2, top panel). With respect to vehicle-treated rats, the amount of self-administered alcohol was reduced by approximately $60 \%$ in all three rat groups treated with GS39783. A reduction of similar magnitude was recorded in the total number of licks on the sipper spout $[F(3,33)=5.82$, $P<0.005$ ] (Figure 2, bottom panel).

\section{DISCUSSION}

The aim of the present study was twofold: first, to evaluate the behavior of selectively bred, alcohol-preferring sP rats when exposed to the so-called "sipper" procedure of operant, oral alcohol self-administration, a procedure thought to better distinguish - compared to other, more conventional procedures between the appetitive and consummatory aspects of animal behavior; second, to assess the effect of GS39783, one of the few $\mathrm{GABA}_{\mathrm{B}}$ PAMs presently available for in vivo testing, on these two aspects of alcohol self-administration behavior.

With regard to the first aim, all tested sP rats acquired and steadily maintained self-administration behavior. Rats displayed a robust motivation to initiate and perform lever-responding for alcohol, as witnessed by short latencies to the first lever-response, high rates of lever-responding (resulting in the achievement of RR55 in 2-3 min), and achievement of an RR as high as 55 . These data are in agreement with previous lines of evidence suggesting that alcohol has strong motivational properties in sP rats (see Colombo et al., 2006). To corroborate this conclusion, it may be helpful to bear in mind that RR55 approximately corresponds to the value of extinction responding (i.e., the total number of unreinforced lever-responding performed in seeking for alcohol) achieved by sP rats trained to self-administer alcohol under the FR4 schedule of reinforcement, and subsequently exposed to a single session of extinction responding (e.g.: Colombo et al., 2003).

Once the alcohol solution was available (upon achievement of RR55), sP rats self-administered large amounts of alcohol (approximately $1.5 \mathrm{~g} / \mathrm{kg}$ ) over a relatively short period of time $(20 \mathrm{~min})$. It may be concluded that the strong motivation of rats to seek alcohol resulted, once alcohol was accessible, in a proportional consumption of alcohol. Notably, the amounts of self-administered alcohol recorded in sP rats exposed to this "sipper" procedure (present study) were markedly larger than those usually recorded in sP rats 


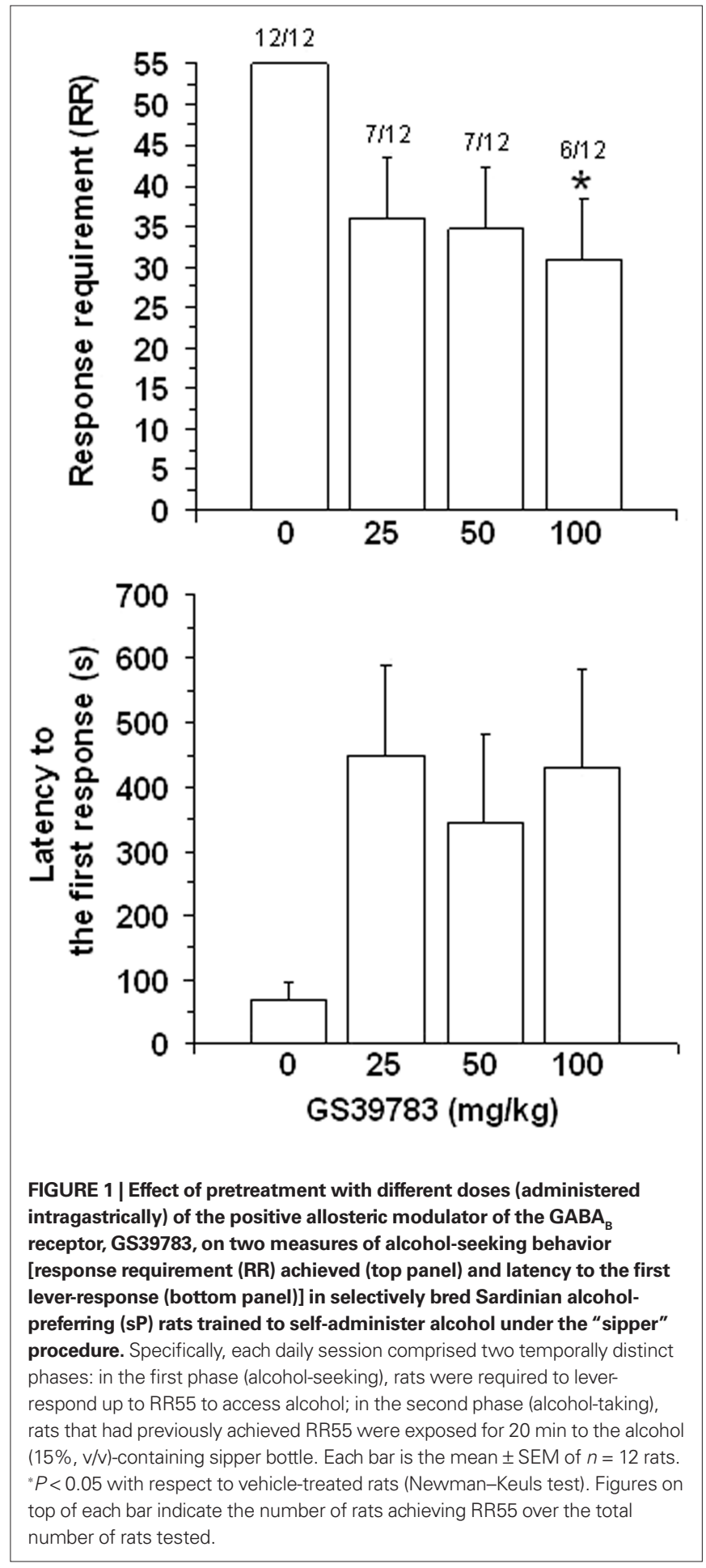

exposed - in daily sessions of comparable length - to the more conventional FR procedure $(0.8-1.0 \mathrm{~g} / \mathrm{kg}$ ) (e.g.: Maccioni et al., $2007,2008,2010)$. These data suggest the power of the "sipper" procedure in triggering high levels of alcohol-seeking and -taking behavior in sP rats.

Pertaining to the second study aim, the collected results indicate that pretreatment with GS39783 inhibited both alcohol-seeking and -taking behavior. With regard to the alcohol-seeking phase,
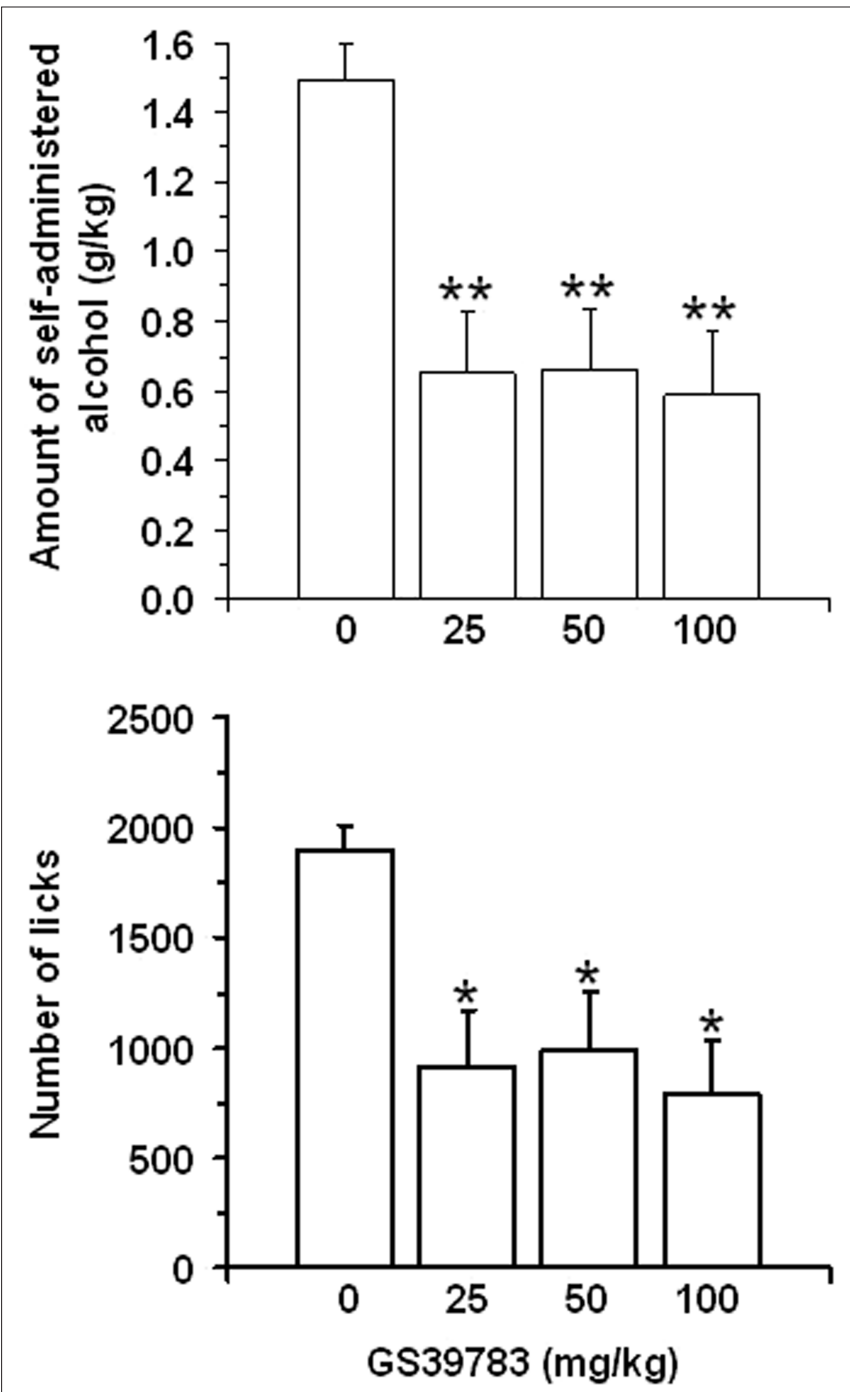

FIGURE 2 | Effect of pretreatment with different doses (administered intragastrically) of the positive allosteric modulator of the $\mathrm{GABA}_{\mathrm{B}}$ receptor, GS39783, on two measures of alcohol-taking behavior [amount of self-administered alcohol (top panel) and number of licks on the sipper spout (bottom panel)] in selectively bred Sardinian alcohol-preferring (sP) rats trained to self-administer alcohol under the "sipper" procedure. Specifically, each daily session comprised two temporally distinct phases: in the first phase (alcohol-seeking), rats were required to lever-respond up to RR55 to access alcohol; in the second phase (alcohol-taking), rats that had previously achieved RR55 were exposed for 20 min to the alcohol (15\%, v/v)-containing sipper bottle. Each bar is the mean \pm SEM of $n=12$ rats. ${ }^{*} P<0.01$ and ${ }^{* *} P<0.001$ with respect to vehicle-treated rats (Newman-Keuls test).

a first line of evidence indicates that virtually half of the rats treated with the three different doses of GS39783 failed to achieve RR55, failing therefore to gain access to alcohol. Notably - among these rats $-3 / 12,2 / 12$, and $3 / 12$ rats of the 25,50 , and $100 \mathrm{mg} / \mathrm{kg}$ GS39783-treated groups even avoided making any response on the lever. In addition, pretreatment with GS39783 was associated with a marked reduction in the mean value of the RR achieved. As observed in previous studies (Maccioni et al., 2007, 2008), the dose-dependence relationship was relatively modest, suggesting 
that wider GS39783 dose-ranges should be tested in future studies. The minimal effect of pretreatment with GS39783 on latency to the first response on the lever is in agreement with previous data collected in self-administration studies conducted with the FR and PR schedules of reinforcement for alcohol (Maccioni et al., 2007, 2008), and confirms that GS39783 may have minimal reducing effect on the motivation to start lever-responding for alcohol.

Pretreatment with GS39783 also affected the alcohol-taking phase, as indicated by a reduction in both the amount of selfadministered alcohol and total number of licks during the 20-min alcohol-taking phase subsequent to achievement of RR55. These data, including the relatively modest dose-dependence relationship of GS39783 effects, are consistent with previous lines of experimental evidence indicating the capacity of GS39783 to reduce the amount of self-administered alcohol in sP rats exposed to the FR and PR schedules of reinforcement (Maccioni et al., 2007, 2008), as well as alcohol intake in sP rats exposed to the two-bottle "alcohol versus water" choice regimen (Orrù et al., 2005).

The present study however features some limitations. Rats were allocated to the different experimental groups (four, one for each tested dose of GS39783) according to a Latin-square design, in which each dose of GS39783 was tested in each rat, with four drugfree, regular self-administration sessions elapsing between test sessions. This design, commonly utilized in pharmacological studies of alcohol self-administration under the FR and PR procedures, had the advantage of dramatically reducing the number of rats to be used ( $n=12$, instead of $n=48$ needed with independent groups of rats). The length of the training procedure, together with the workload and equipment required, would have made the use of independent rat groups unfeasible. Statistical analysis of data generated by a Latin-square design requires the use of repeated measures, as all rats undergo all drug treatments. In the case of the present study, the Latin-square design implied the disadvantage, or limitation, that a value of latency, alcohol consumption, and number of licks had to be assigned to rats that did not press the lever or did not achieve RR55. Specifically, a latency value of $1200 \mathrm{~s}$ was assigned to rats failing to press the lever; values equal to $0 \mathrm{~g} / \mathrm{kg}$ alcohol and 0 licks were assigned to rats not achieving RR55 and, therefore, not gaining access to alcohol. Assignment of these arbitrary values may have amplified the differences between vehicle- and GS39783treated rats. However, it may be noteworthy that a reduction (by approximately 25\%) in the amount of self-administered alcohol

\section{REFERENCES}

Colombo, G., Lobina, C., Carai, M.A. M., and Gessa, G. L. (2006). Phenotypic characterization of genetically selected Sardinian alcohol-preferring $(\mathrm{sP})$ and -non preferring (sNP) rats. Addict. Biol. 11, 324-338.

Colombo, G., Vacca, G., Serra, S., Brunetti, G., Carai, M. A. M., and Gessa, G. L. (2003). Baclofen suppresses motivation to consume alcohol in rats. Psychopharmacology (Berl.) 167, 221-224.

Cryan, J. F., Kelly, P. H., Chaperon, F., Gentsch, C., Mombereau, C.,
Lingenhoehl, K., Froestl, W., Bettler, B., Kaupmann, K., and Spooren, W. P. (2004). Behavioral characterization of the novel GABAB receptor-positive modulator GS39783 (N,N'-dicyclopentyl-2methylsulfanyl-5-nitro-pyrimidine4,6-diamine): anxiolytic-like activity without side effects associated with baclofen or benzodiazepines. J. Pharmacol. Exp. Ther. 310, 952-963.

Czachowski, C. L., Legg, B. H., and Samson, H. H. (2001). Effects of acamprosate on ethanol-seeking and

and total number of licks was still recorded between vehicle-treated rats and the GS39783-treated rats that achieved RR55 and accessed alcohol; because of the lack of statistical analysis, this observation may be considered suggestive and not conclusive.

A second limitation of the present study was the lack of investigation on the specificity of GS39783 on alcohol self-administration. In pharmacological studies, the specificity of the effect of the tested drug on alcohol self-administration is commonly assessed by evaluation of the effect of the drug on self-administration for an alternative, non-drug reinforcer (e.g., a sucrose or saccharin solution or food pellets). Based on the results of previous FR and PR studies (Maccioni et al., 2007, 2008) - demonstrating that administration to sP rats of $25-100 \mathrm{mg} / \mathrm{kg}$ GS39783 was totally devoid of any effect on self-administration of sucrose solutions the reinforcing and motivational properties of which were identical to those of $15 \%$ alcohol - it was assumed that it would be highly likely that the reducing effect of GS39783 observed in the present study was specific for alcohol.

The lack of any GS39783-induced alteration of sucrose selfadministration observed in the two previous studies (Maccioni et al., 2007, 2008) is of interest also as it tends to exclude that the reducing effect of this dose-range of GS39783 on alcohol selfadministration may be secondary to possible motor-incapacitating or sedative effects (conceivable because of the drug activity on GABAergic neurotransmission), resulting in a disruption of normal rates of lever-responding.

In conclusion, the results of the present study indicate that the "sipper" procedure of alcohol self-administration (based on two temporally distinct phases of alcohol-seeking and -taking behavior) is extremely effective in inducing high levels of lever-responding and alcohol consumption in alcohol-preferring sP rats. Further, the results of the present study extend to this self-administration procedure the capacity of the $\mathrm{GABA}_{\mathrm{B}} \mathrm{PAM}, \mathrm{GS} 39783$, to reduce the appetitive, or motivational, properties of alcohol and alcohol consumption in $\mathrm{sP}$ rats.

\section{ACKNOWLEDGMENTS}

The authors are grateful to Mrs. Carla Acciaro for animal breeding and care, and Ms. Anne Farmer for language editing of the manuscript. This study was partially supported by a grant from Compagnia di San Paolo - Programma Neuroscienze 2008/09, Turin, Italy.

self-administration in the rat. Alcohol. Clin. Exp. Res. 25, 344-350.

Czachowski, C. L., Legg, B. H., and Stansfield, K. H. (2006). Ethanol and sucrose seeking and consumption following repeated administration of the GABA(B) agonist baclofen in rats. Alcohol. Clin. Exp. Res. 30, 812-818.

Freedland, C. S., Sharpe, A. L., Samson, H.H., and Porrino, L. J. (2001). Effects of SR141716A on ethanol and sucrose self-administration. Alcohol. Clin. Exp. Res. 25, 277-282.

Leggio, L., Garbutt, J. C., and Addolorato, G. (2010). Effectiveness and safety of baclofen in the treatment of alcohol dependent patients. CNS Neurol. Disord. Drug Targets 9, 33-44.

Liang, J. H., Chen, F., Krstew, E., Cowen, M. S., Carroll, F. Y., Crawford, D., Beart, P. M., and Lawrence, A. J. (2006). The GABAB receptor allosteric modulator CGP7930, like baclofen, reduces operant self-administration of ethanol in alcohol-preferring rats. Neuropharmacology 50, 632-639.

Maccioni, P., Carai, M. A. M., Kaupmann, K., Guery, S., Froestl, W., Leite-Morris, K. A., Gessa, G. L., and Colombo G. (2009). 
Reduction of alcohol's reinforcing and motivational properties by the positive allosteric modulator of the GABAB receptor, BHF177, in alcohol-preferring rats. Alcohol. Clin. Exp. Res. 33, 1749-1756.

Maccioni, P., and Colombo, G. (2009). Role of the GABAB receptor in alcohol seeking and drinking behavior. Alcohol 43, 555-558.

Maccioni, P., Fantini, N., Froestl, W., Carai, M.A.M., Gessa, G. L., and Colombo G. (2008). Specific reduction of alcohol's motivational properties by the positive allosteric modulator of the GABAB receptor, GS39783 - Comparison with the effect of the $\mathrm{GABAB}$ receptor direct agonist, baclofen. Alcohol. Clin. Exp. Res. 32, 1558-1564.

Maccioni, P., Pes, D., Orrù, A., Froestl, W., Gessa, G. L., Carai, M. A. M., and Colombo, G. (2007). Reducing effect of the positive allosteric modulator of the GABAB receptor, GS39783, on alcohol self-administration in alcohol-preferring rats.
Psychopharmacology (Berl.) 193, 171-178.

Maccioni, P., Thomas, A. W., Carai, M. A. M., Gessa, G. L., Malherbe, P., and Colombo, G. (2010). The positive allosteric modulator of the GABAB receptor, rac-BHFF, suppresses alcohol self-administration. Drug Alcohol Depend. 109, 96-103.

Markou, A., Weiss, F., Gold, L. H., Caine, S. B., Schulteis, G., and Koob, G. F. (1993). Animal models of drug craving. Psychopharmacology (Berl.) 112, 163-182.

Orrù, A., Lai, P., Lobina, C., Maccioni, P., Piras, P., Scanu, L., Froestl, W., Gessa, G. L., Carai, M. A. M., and Colombo, G. (2005). Reducing effect of the positive allosteric modulators of the GABAB receptor, CGP7930 and GS39783, on alcohol intake in alcohol-preferring rats. Eur. J. Pharmacol. 525, 105-111.

Samson, H. H., Czachowski, C. L., and Slawecki, C. J. (2000). A new assessment of the ability of oral ethanol to function as a reinforcing stimulus. Alcohol. Clin. Exp. Res. 24, 766-773.

Sharpe, A. L., and Samson, H. H. (2002). Repeated nicotine injections decrease operant ethanol self-administration. Alcohol 28, 1-7.

Slawecki, C. J., and Roth, J. (2003). Neurokinin type- 3 receptor stimulation impairs ethanol-associated appetitive behavior in Wistar rats. Alcohol. Clin. Exp. Res. 27, 1962-1970.

Urwyler, S., Pozza, M. F., Lingenhoehl, K., Mosbacher, J., Lampert, C., Froestl, W., Koller, M., and Kaupmann, K. (2003). N,N'-Dicyclopentyl-2methylsulfanyl-5-nitro-pyrimidine4,6-diamine (GS39783) and structurally related compounds: novel allosteric enhancers of gamma-aminobutyric acidB receptor function. J. Pharmacol. Exp. Ther. 307, 322-330.

Conflict of Interest Statement: The authors declare that the research was conducted in the absence of any commercial or financial relationships that could be construed as a potential conflict of interest.

Received:01 June 2010; paperpending published: 15 June 2010; accepted: 21 June 2010; published online: 19 July 2010.

Citation: Maccioni P, Flore P, Carai MAM, Mugnaini C, Pasquini S, Corelli F, Gessa GL and Colombo G (2010) Reduction by the positive allosteric modulator of the $G A B A_{B}$ receptor, GS39783, of alcohol self-administration in Sardinian alcohol-preferring rats exposed to the "sipper" procedure. Front. Psychiatry 1:20. doi: 10.3389/fpsyt.2010.00020

This article was submitted to Frontiers in Addictive Disorders, a specialty of Frontiers in Psychiatry.

Copyright (C) 2010 Maccioni, Flore, Carai, Mugnaini, Pasquini, Corelli, Gessa and Colombo. This is an open-access article subject to an exclusive license agreement between the authors and the Frontiers Research Foundation, which permits unrestricted use, distribution, and reproduction in any medium, provided the original authors and source are credited. 